\title{
Long non-coding NEAT1 weakens the protective role of sevoflurane on myocardial ischemia/reperfusion injury by mediating the microRNA-140/RhoA axis
}

\section{Pengfei Rui}

Liyang People's Hospital of Jiangsu Province

Junhua Wang

Liyang People's Hospital of Jiangsu Province

Jing Xu ( $\square$ Xujing010905@163.com )

Liyang People's Hospital of Jiangsu Province https://orcid.org/0000-0003-4936-2902

\section{Research article}

Keywords: Sevoflurane, Long non-coding RNA NEAT1, microRNA-140, RhoA, Myocardial ischemia/reperfusion

Posted Date: June 10th, 2020

DOI: https://doi.org/10.21203/rs.3.rs-32194/v1

License: (c) (i) This work is licensed under a Creative Commons Attribution 4.0 International License.

Read Full License 


\section{Abstract}

Background: The inhaled Sevoflurane (Sev) has been implicated to protect myocardial tissues against ischemia/reperfusion (I/R)-evoked injury. Nevertheless, the detailed mechanisms of Sev-mediated cardioprotection remain basically unknown. This study intends to examine the roles of nuclear enriched abundant transcript 1 (NEAT1), a long non-coding RNA (IncRNA) during the cardioprotection exerted by Sev.

Methods: We initially performed I/R surgery in mice, and Sev treatment was given during the perfusion, followed by the determination of cardiac function, myocardial infarction area and myocardial apoptosis for the validation of the model. Then, we conducted IncRNA microarray analysis on Sev-treated hearts to find out IncRNAs with significant differences in expression. The changes of myocardial I/R injury were evaluated by echocardiography, TTC staining and TUNEL assay after establishing mice with NEAT1 upregulation. Afterwards, the targeting miRNA of NEAT1 and the targeting mRNA of the miRNA were screened out through StarBase and microarray analyses to carry out rescue experiments.

Results: NEAT1 was downregulated in Sev-treated cardiomyocytes. Overexpression of NEAT1 weakened cardiac function, increased infarct size and increased apoptosis in the presence of Sev. NEAT1 bound to microRNA (miR)-140, which was significantly upregulated in Sev-treated cardiomyocytes. Inhibition of miR-140 expression weakened the cardiac function of Sev-treated mice, increased infarct size and cardiomyocyte apoptosis. miR-140 mediated the RhoA pathway, and the RhoA activity inhibition attenuated miR-140 effects on Sev protection.

Conclusion: Sev mitigates myocardial I/R injury by restoring NEAT1 expression and regulating the miR140/RhoA axis.

\section{Background}

Ischemia/reperfusion (I/R) is characterized by deficiency of oxygen supply and the following restoration of blood flow, resulting in irreversible injuries to tissues [1]. Acute myocardial infarction is one of the leading causes of death and disability in the world range, and the treatment option for easing myocardial injury and reducing myocardial infarction size is timely and operative reperfusion using thrombolytic therapies or primary percutaneous coronary intervention [2]. Sevoflurane (Sev) is a volatile anesthetic agent that offers hypnosis, analgesia and akinesia during surgical and procedural interventions [3]. The role of Sev during I/R injury has been underscored in the lung [4], brain [5] as well as heart [6].

Interestingly, LINC00652, a kind of long non-coding RNA (IncRNA), was observed to work with Sev to exert cardioprotective role against myocardial I/R injury [7]. Hence, we postulated that IncRNAs may engage in the Sev-mediated myocardial I/R injury alleviation.

Noncoding RNAs, a group of RNAs that do not encode proteins, can be divided into IncRNAs, microRNAs (miRNAs) as well as circular RNAs and are closely related to cardiovascular disease by regulating cell proliferation, apoptosis and other vital biological events [8]. For example, downregulation of nuclear 
enriched abundant transcript 1 (NEAT1) acted as a protector against the apoptosis of cardiomyocytes under hypoxia/reoxygenation [9]. It has been proposed that competing endogenous RNAs (ceRNAs) modulate the distribution of miRNAs on their target mRNAs, thereby exerting a post-transcriptional regulation [10]. For instance, NEAT1 enhanced myocardial I/R injury by competing with mitogen-activated protein kinase 6 to regulate miR-495-3p [11]. While miR-140, which is predicted a binding miRNA of NEAT1 by a bioinformatics website in the current study, was found to attenuate myocardial I/R injury via inhibiting mitochondria-mediated apoptosis by interacting with YES1 [12]. Also, IncRNA SNHG1 protected SH-SY5Y cells from injury caused by oxygen glucose deprivation through the miR-140-5p/Bcl-XL axis [13]. The additional activity of Ras-associated guanosine triphosphatase RhoA is monitored to promote oxidative stress and the progression of cardiovascular diseases [14]. More specifically, the protective role of Ginsenoside Rb1 against I/R-induced myocardial injury was achieved by mediating the RhoA signaling pathway [15]. In addition, RhoA was found to share binding sites with miR-140 in the present study by Starbase. Based on the aforementioned reports, we carried out this experiment to probed the function of NEAT1 during injury evoked by myocardial I/R in a developed mouse model by regulating the miR$140 /$ RhoA axis with the administration of Sev.

\section{Methods}

\section{Mouse treatment}

Our experiments were carried out under the approval of the Ethics Committee of Liyang People's Hospital of Jiangsu Province on the basis of the Guide for the Care and Use of Laboratory Animals formulated by the National Institutes of Health. Substantial efforts were made to minimize the suffering and numbers of the animals used during the experiment. All mice used in this study were from Beijing Vital River Laboratory Animal Technology Co., Ltd. (Beijing, China). Twenty-seven C57BL/6N male mice (aged 6-8 weeks, weight $15-25 \mathrm{~g}$ ) were allowed to acclimatize for one week in the animal facility (at a constant temperature of $22^{\circ} \mathrm{C}$ with $12 \mathrm{~h}$ light and dark cycles) before any intervention was initiated. Mice were allocated into sham (shamed-operated mice), I/R (mice subjected to I/R surgery only), I/S (mice inhaled with Sev during the reperfusion of I/R surgery), NEAT1-negative control (NC) (mice injected with mock 48 $\mathrm{h}$ before I/R treatment), NEAT1-overexpression (OE, mice injected with NEAT1-OE $48 \mathrm{~h}$ before I/R treatment), miR-140 control (mice injected with miR-mock $48 \mathrm{~h}$ before l/R treatment), miR-140 inhibitor (mice injected with miR-140 inhibitor $48 \mathrm{~h}$ before I/R treatment), miR-140 inhibitor + dimethylsulfoxide (DMSO, mice injected with miR-140 inhibitor and DMSO $48 \mathrm{~h}$ before I/R treatment), miR-140 inhibitor + C3 (mice injected with miR-140 inhibitor and extracellular enzyme C3 $48 \mathrm{~h}$ before l/R treatment) groups. NEAT1-OE and miR-140 inhibitor fragments and NCs were synthesized by GenePharma (Shanghai, China). The lentiviral vector pLenti6 (Thermo Fisher Scientific Inc., Waltham, MA, USA) was transfected with RNA fragments by BLOCK-iT lentiviral RNAi expression system (Thermo Fisher Scientific). The mice were then anesthetized with $1 \%$ pentobarbital sodium through an intraperitoneal injection at $60 \mathrm{mg} / \mathrm{kg}$. A longitudinal incision was made between the $2^{\text {nd }}, 3^{\text {rd }}$ and $4^{\text {th }}$ ribs to expose the heart. The corresponding 
transfection solution $(10 \mu \mathrm{L})$ was slowly injected at the anterior wall of the left ventricle of the mouse. After the injection, the mouse chest was sutured.

\section{Development of a myocardial I/R mouse model}

On the day of I/R experiment, all mice were injected intraperitoneally with atropine at $0.04 \mathrm{mg} / \mathrm{kg}$ and with pentobarbital sodium at $60 \mathrm{mg} / \mathrm{kg}$ after $5 \mathrm{~min}$. Once the respiratory frequency of mice reached 90 times/min, the mice were connected to an animal breathing apparatus, which was set at the frequency 90-105 times/min with a tidal volume at $\mathrm{mL}$. A longitudinal incision was made between the second and fourth ribs to expose the heart and pericardium. The left anterior descending coronary artery was ligated from the tip of the left auricle. An electrocardiogram (ECG) was applied to assess the ischemic condition. After $30 \mathrm{~min}$, blood flow was restored for reperfusion for $15 \mathrm{~min}$, during which mice in the IS groups were inhaled with $2.4 \%$ Sev continuously, and mice in the sham group was inhaled with common air. The intercostal and pectoral muscles were sutured to close the chest after heart rhythm stabilization. After the suture, excess gas was extracted with needles from thoracic cavities. When the mice had mild spontaneous respiration, the apparatus was removed. Following the skin was sutured, the mice were put back into $25^{\circ} \mathrm{C}$ animal rooms with a $0.5 \mathrm{~mL}$ saline intraperitoneal injection.

\section{ECG}

A small animal ultrasound imaging system was purchased from VisualSonics (FUJIFILM VisualSonics, Inc., Toronto, ON, Canada). The left ventricular end-diastolic volume (LVEDV), left ventricular end-systolic volume (LVESV), left ventricular end-diastolic diameter (LVDd) and left ventricular end-systolic diameter (LVDs) were measured to obtain left ventricular ejection fraction (LVEF) and left ventricular fractional shortening $($ LVFS). LVEF $(\%)=($ LVEDV - LVESV $) /($ LVEDV $) \times 100 \%$, LVFS $(\%)=($ LVDd - LVDs $) / L V D d \times$ $100 \%$.

\section{Triphenyl tetrazolium chloride (TTC) staining}

After testing the cardiac cycle of mice, we performed euthanasia by intraperitoneal injection of $1 \%$ sodium pentobarbital at $150 \mathrm{mg} / \mathrm{kg}$. Once observing the lack of heartbeat, respiratory arrest, pupil dilation, and lack of nerve reflex, the mouse heart tissues were removed and fixed in $4 \%$ paraformaldehyde immediately, frozen and cut into $5 \mu \mathrm{m}$ slices. Afterwards, sliced fresh tissues were cultured in a petri dish containing TTC staining solution (Solabio, China). After a 30-min incubation at $37^{\circ} \mathrm{C}$, the stained sections were removed with a pipette, rinsed with $4^{\circ} \mathrm{C}$ phosphate buffered saline, observed by naked eyes and photographed. The red part represents normal non-ischemic myocardial tissues, the gray part represents ischemic but non-infarct myocardial tissues, and the white part represents myocardial tissues with ischemic infarction. Image-Pro Plus 6.0 software was applied to determine the percentage of myocardial infarction area.

\section{Terminal deoxynucleotidyl transferase (TdT)-mediated dUTP nick-end labeling (TUNEL) staining}


The cells in logarithmic growth of each group were plated at $1 \times 10^{6}$ cells $/ \mathrm{mL}$ on the cover glasses in the 6-well plate. TUNEL apoptosis was detected using In Situ Cell Death Detection Kit-peroxidase (Roche, Basel, Switzerland). The cell suspension was coated on the cover glasses, reacted with Proteinase K for $15 \mathrm{~min}$ and incubated with $50 \mu \mathrm{L}$ TUNEL solution in a dark chamber for $1 \mathrm{~h}$ at $37^{\circ} \mathrm{C}$. Following a 10 -min treatment with $50 \mu \mathrm{L}$ diaminobenzidine, the results were recorded using an M-60 microscope (Olympus Optical Co., Ltd., Tokyo, Japan) equipped with $\mathrm{BH} 2$ system with attached $35 \mathrm{~mm}$ camera (Olympus). The number of TUNEL-positive cells was counted under five randomly-selected high magnification views.

\section{Microarray analysis}

Total RNA was extracted by ice-lysed mouse tissue homogenate in Trizol (Beijing Solabio Life Sciences Co., Ltd., Beijing, China). A total of $0.5 \mu \mathrm{g}$ total RNA was reversely transcribed into cDNA through PrimeScript ${ }^{\text {TM }}$ IV 1st strand cDNA Synthesis Mix (Takara Holdings Inc., Kyoto, Japan). The cDNA was then fragmented and hybridized with a mouse IncRNA expression array V6.0 (Shanghai Biotechnology Corporation), and then the array was screened using GeneChip ${ }^{\mathrm{TM}}$ Scanner $30007 \mathrm{G}$ system (Thermo Fisher Scientific Inc., Waltham, MA, USA). Scanning results were subjected to quality assessment and data correction. $\mathrm{R}$ language package was used to plot the heatmap using processed data. $p<0.01$ and $\| \log _{2}$ FoldChangel were used as the screening criteria, and the top ten RNAs were selected for analysis.

\section{Reverse transcription quantitative polymerase chain reaction (RT-qPCR)}

Total RNA was used for RNA extraction from tissues using Trizol. Totally $5 \mu$ total RNA was collected for reverse transcription and RT-qPCR through the SuperScript ${ }^{\text {TM }}$ IV One-Step RT-PCR System. U6 and glyceraldehyde-3-phosphate dehydrogenase (GAPDH) served as the internal normalized references. The primers are displayed in Table 1. Relative expression of targets was determined using $2-\Delta \Delta \mathrm{Ct}$.

\section{Luciferase reporter assay}

HEK293T cells (Cell Bank of Shanghai Institute of Cells, Chinese Academy of Science, Shanghai, China) were grown in Dulbecco's modified Eagle's medium. When the density reached $80 \%-90 \%$, the cells were detached with $0.25 \%$ trypsin and passaged in a $5 \% \mathrm{CO}_{2}$ incubator at $37^{\circ} \mathrm{C}$, and the cells in logarithmic growth phase were harvested for further experiments. The targets of NEAT1 and miR-140 were analyzed using StarBase and verified by luciferase reporter assays. The miRNA 3'untranslated region (3'UTR) sequence and the IncRNA and mRNA 3'UTR sequence were inserted into pGL4.18 luciferase reporter vectors (Promega Corporation). HEK293T cell co-transfection was carried out using Lipofectamine 3000 (Invitrogen, Carlsbad, CA, USA). After $24 \mathrm{~h}$, the cells were lysed, and the luciferase activity was assessed by a dual-luciferase reporter assay system (Promega). The ratio of firefly luciferase activity to renilla luciferase activity reflects the relative luciferase activity.

\section{Western blot}


After $48 \mathrm{~h}$ of transfection, the cells were lysed on ice with radio immunoprecipitation assay lysis buffer. The samples were centrifuged at $20000 \mathrm{~g}$ for a period of $10 \mathrm{~min}$ at $4^{\circ} \mathrm{C}$ to collect the supernatant. Following the protein quantification using a bicinchoninic acid kit (Sigma-Aldrich Chemical Company, St Louis, MO, USA). We then separated $15 \mu$ g protein lysates using $12 \%$ sodium dodecyl sulphatepolyacrylamide gel electrophoresis. Afterwards, the proteins were transferred to polyvinylidene fluoride membranes (Millipore, Billerica, MA, USA) and sealed with $5 \%$ skim milk for $2 \mathrm{~h}$. Blots were probed with the primary antibody against RhoA (ab187027, Abcam Inc., Cambridge, UK) at $4^{\circ} \mathrm{C}$ overnight and with the secondary antibody (ab205718, Abcam) for $3 \mathrm{~h}$ at room temperature. At last, the immunoblot was visualized by Superstar electrochemiluminescence Plus. Gel imaging system (Bio-Rad Laboratories, Hercules, CA, USA) was applied to determine protein expression.

\section{Statistics}

Statistical analysis was validated by SPSS 22.0 (IBM Corp. Armonk, NY, USA). All data are expressed as mean \pm standard derivation (SD). Statistical significance was determined using unpaired $t$ test and oneway or two-way analysis of variance (ANOVA), as appropriate. If ANOVA was significant, individual differences were assessed using the Tukey's post-test. For all studies, values of $p<0.05$ were considered statistically significant.

\section{Results}

\section{Reduction of myocardial I/R injury is found in mice treated with Sev}

To test whether the injury following I/R was successfully induced and the relevance of Sev in the biochemical parameters related to cardiac function were measured. The LVEF and LVFS of I/R-treated mice were lower than that of sham-operated mice, and the those of Sev-treated mice were higher than that of the I/R-treated mice without inhalation (Figure 1A). We later found that the myocardial infarction area of Sev-treated mice was significantly smaller than that of mice subjected to I/R alone by heart observation (Figure 1B). Subsequently, it was further exhibited that the apoptosis of cardiomyocytes in mice after Sev treatment was significantly reduced (Figure 1C), which implied that the I/R model was effectively developed and that Sev treatment could reduce the occurrence of injury caused by myocardial $\mathrm{I} / \mathrm{R}$ in mice.

\section{Decreased NEAT1 expression is identified in mice with myocardial I/R following Sev treatment}

We then performed a IncRNA microarray analysis on myocardial tissues of I/R mice treated with Sev or without any other treatment. The microarray analysis results showed that NEAT1 was significantly downregulated after Sev inhalation (Figure 2A), which was verified by RT-qPCR (Figure 2B). We then injected NEAT1-NC and NEAT1-OE into the nude mice, and the success of model construction was established by RT-qPCR analysis of cardiomyocytes (Figure 2C).

NEAT1 inhibits the role of Sev in myocardial I/R injury 
The LVEF and LVFS indices of NEAT1-OE-injected mice were notably decreased versus compared with NEAT1-NC-injected mice (Figure 3A). Meanwhile, NEAT1-OE increased significantly the myocardial infarction area of the heart compared to the NEAT1-NC (Figure 3B), and overexpression of NEAT1 also resulted in an increased rate of apoptosis in mouse cardiomyocytes (Figure $3 \mathrm{C}$ ). Here it can be inferred that NEAT1 could reduce the alleviating role of Sev in injury caused by myocardial I/R.

\section{miR-140 promotes the role of Sev in myocardial I/R injury}

We then performed a miRNA microarray analysis on myocardial tissues of I/R mice treated with Sev or without any other treatment to screen the significantly upregulated miRNAs (Figure 4A), which was intersected with the binding miRNAs of NEAT1 predicted by StarBase (Figure 4B). miR-140 was remarkably upregulated in I/R mice treated with Sev and had a targeting relationship with NEAT1. The results of dual-luciferase reporter gene assays showed that in terms of the miR-140 mimic group, the luciferase activity of NEAT1-wild type (WT) was profoundly lower than that of the miR-140 NC group (Figure 4C). RT-qPCR validated that miR-140 was significantly upregulated in mice subjected to I/R after Sev treatment (Figure 4D). Then we analyzed the expression of miR-140 in mice injected with NEAT1-NC or NEAT1-OE and found that the miR-140 expression in NEAT1-OE-injected mice was decreased (Figure $4 \mathrm{E})$. As a consequence, we administrated miR-140 inhibitor or control into nude mice, which was verified by RT-qPCR (Figure 4F). miR-140 inhibitor resulted in significantly lower LVEF and LVFS indices than the miR-140 control (Figure 4G). Meanwhile, miR-140 inhibitor led to increased myocardial infarction area in the mouse heart compared with miR-140 control (Figure 4H). Poor expression of miR-140 also caused increased apoptosis rate in mouse cardiomyocytes (Figure 4I).

\section{The inhibition of RhoA partially restores the effect of miR-140 on Sev}

StarBase predicted that RhoA was a possible target of miR-140, which was further corroborated by dualluciferase reporter assays (Figure 5A). The expression of RhoA in mice injected with NEAT1-NC, NEAT1OE, miR-140 control or miR-140 inhibitor was analyzed, and its expression was increased in mice with NEAT1-OE and miR-140 inhibitor (Figure 5B). To further verify the effect of RhoA on I/R injury, we treated mice with RhoA inhibitor Exoenzyme C3 or DMSO in the presence of miR-140 inhibitor, and the success of which was authenticated by western blot analysis (Figure 5C). In mice treated with miR-140 inhibitor + C3, the LVEF and LVFS were increased (Figure 5D), myocardial infarction area was diminished (Figure 5E) and the apoptosis rate of mouse cardiomyocytes (Figure 5F) was also decreased significantly relative to the miR-140 inhibitor + DMSO treatment. This proves that the weakening of RhoA activity partially restored the effect of miR-140 on Sev.

\section{Discussion}

In spite of the fact that substantial advances have been made in antithrombotic therapy, the mortality of patients with myocardial I/R injury remains dismal; consequently, it is an urgency to improve the efficacy of cardioprotective agents to ameliorate myocardial function and limit the total area of infarction during injury caused by I/R [16]. Besides, the emerging relevance of IncRNAs in I/R injury of heart caught our 
attention [17]. In view of this, we performed this study to decipher the potential mechanism behind myocardial I/R injury, and our obtained data indicate that poor expression of NEAT1 is able to attenuating myocardial I/R injury after Sev treatment in mice by mediating the miR-140/RhoA axis.

Excessive apoptosis is associated with myocardial ischemia, I/R injury as well as postischemia cardiac remodeling [18], while infarct size is an independent indicator effective in predicting mortality after infarction [19]. In the current work, we observed that Sev worked as a cardio-protector against injury induced by myocardial I/R by reducing LVEF, LVFS, myocardial infarct size and apoptosis rate, indicating the beneficial role of Sev on I/R injury [20,21]. Moreover, Sev was found to diminish NEAT1, which was enhanced in mice with myocardial I/R, by microarray analysis. NEAT1 has been most frequently studied in various cancer as an oncogene, including cervical cancer [22], colon cancer [23] as well as glioma [24] by regulating cell migration, proliferation along with drug resistance. Additionally, NEAT1 was upregulated in Parkinson's disease and conferred drug-inducible neuroprotection against oxidative stress [25]. Also, NEAT1 expression was enhanced in patients suffered from acute ischemic stroke versus controls, which could predict unsatisfactory recurrence-free survival [26]. Furthermore, NEAT1 overexpression repressed the alleviating role of Sev in mice with injury induced by myocardial I/R. Consistently, NEAT1 was overexpressed in cells with myocardial I/R injury relative to normal myocardial cells, and declines in NEAT1 expression contributed to enhanced cell proliferation and inhibited cell apoptosis [27]. Meanwhile, silencing of NEAT1 promoted LVEF and LVFS to normal levels, implying a protective role of NEAT1 knockdown in diabetic rats with myocardial I/R injuries [28].

The relevance of IncRNA-miRNA-mRNA network in cardiovascular diseases has been highlighted recently [29]. After determination of the stimulative role of NEAT1 on myocardial I/R injury, we next sought to its target miRNA. Through a miRNA microarray analysis of significantly upregulated miRNAs in mice after Sev and Starbase prediction, miR-140 was substantiated as our target. In line with our finding, miR-370 was reduced in mice exhibited IR injuries, but Sev anesthetic preconditioning promoted the miR-370 expression pattern [30]. Previously, circRNA_000203 aggravated cardiac hypertrophy by lowering the expression of miR-140-3p [31]. Additionally, miR-140-5p was corroborated as a promising therapeutic target for alleviating brain injury induced by intracerebral hemorrhage [32]. Our data presented that with the treatment of miR-140 inhibitor, the effects of Sev were inhibited. Moreover, Catalpol improved myocardial damage by regulating NEAT 1/miR-140-5p in mice with diabetic cardiomyopathy in vivo [33]. Lastly, we screened out RhoA as a putative target of miR-140. Besides, RhoA shares a positive correlation with NEAT1 and an inverse one with miR-140. RhoA activity in myocardium of the Sprague-Dawley rats $(0.35 \pm 0.06)$ enhanced remarkably compared with that in the sham-operated rats $(0.18 \pm 0.03)$ [34]. Suppression of RhoA prevented propofol-regulated hippocampal neurotoxicity and following cognitive deficits [35]. The TLR4/RhoA/ROCK pathway was indicated in blood-brain barrier impairment after cerebral I/R injury [36]. Furthermore, Forgione et al. showed that C3 transferase, which could selectively inhibit Rho expression without disturbing other guanine triphosphatases, has the potency to augment neuroprotection and axonal regeneration following spinal cord injury [37]. Nevertheless, the cardioprotection role of exoenzyme C3 was rarely probed. Here we reported that $\mathrm{C} 3$ could rescue the inhibitory role of miR-140 on Sev-mediated injury alleviation in mice. 


\section{Conclusion}

Overall, the present study established that NEAT1 positively regulates RhoA expression by binding to miR140 , by which the mechanism of action of the cardioprotection of Sev is weakened against myocardial $\mathrm{I} / \mathrm{R}$ in the mouse model (Figure 6). NEAT1 silencing may act as an attractive target for promoting Sevinduced myocardial protection. Further works are warranted to obtain more information about the NEAT1/miR-140/RhoA axis in vitro, and to explore new treatment options for myocardial I/R injury.

\section{Abbreviations}

ceRNAs, competing endogenous RNAs; DMSO, dimethylsulfoxide; ECG, electrocardiogram; GAPDH, glyceraldehyde-3-phosphate dehydrogenase; I/R, ischemia/reperfusion; IncRNA, long non-coding RNA; LVDd, left ventricular end-diastolic diameter; LVDs, left ventricular end-systolic diameter; LVEDV, left ventricular end-diastolic volume; LVEF, left ventricular ejection fraction; LVESV, left ventricular end-systolic volume; LVFS, left ventricular fractional shortening; miR, microRNA; NC, negative control; NEAT1, nuclear enriched abundant transcript 1; OE, overexpression; RT-qPCR, reverse transcription quantitative polymerase chain reaction; SD, standard derivation; Sev, Sevoflurane; TTC, 2,3,5-Triphenyl tetrazolium chloride; TUNEL, terminal deoxynucleotidyl transferase (TdT)-mediated dUTP nick-end labeling; 3'UTR, 3'untranslated region;

\section{Declarations}

\section{Ethics approval and consent to participate}

Our experiments were carried out under the approval of the Ethics Committee of Liyang People's Hospital of Jiangsu Province on the basis of the Guide for the Care and Use of Laboratory Animals formulated by the National Institutes of Health. Substantial efforts were made to minimize the suffering and numbers of the animals used during the experiment.

\section{Consent for publication}

Not applicable.

\section{Availability of data and materials}

The datasets used and/or analysed during the current study available from the corresponding author on reasonable request.

\section{Competing interests}

All authors declare no conflict of interest.

\section{Funding}


Not applicable.

\section{Authors' contributions}

PFR designed the experiments. PFR, JHW and JX performed each of the tests and collated the data. All authors analysed the results and prepared the manuscript and they all read and approved the manuscript.

\section{Acknowledgements}

Not applicable.

\section{References}

1. Lejay A, Fang F, John R, Van JA, Barr M, Thaveau F, Chakfe N, Geny B, Scholey JW: Ischemia reperfusion injury, ischemic conditioning and diabetes mellitus. J Mol Cell Cardiol 2016, 91:11-22.

2. Hausenloy DJ, Yellon DM: Myocardial ischemia-reperfusion injury: a neglected therapeutic target. J Clin Invest 2013, 123(1):92-100.

3. Edgington TL, Maani CV: Sevoflurane. In: StatPearls. edn. Treasure Island (FL); 2020.

4. Rancan L, Huerta L, Cusati G, Erquicia I, Isea J, Paredes SD, Garcia C, Garutti I, Simon C, Vara E: Sevoflurane prevents liver inflammatory response induced by lung ischemia-reperfusion. Transplantation 2014, 98(11):1151-1157.

5. Wen XR, Fu YY, Liu HZ, Wu J, Shao XP, Zhang XB, Tang M, Shi Y, Ma K, Zhang F et al: Neuroprotection of Sevoflurane Against Ischemia/Reperfusion-Induced Brain Injury Through Inhibiting JNK3/Caspase-3 by Enhancing Akt Signaling Pathway. Mol Neurobiol 2016, 53(3):1661-1671.

6. Zhao J, Wang F, Zhang Y, Jiao L, Lau WB, Wang L, Liu B, Gao E, Koch WJ, Ma XL et al: Sevoflurane preconditioning attenuates myocardial ischemia/reperfusion injury via caveolin-3-dependent cyclooxygenase-2 inhibition. Circulation 2013, 128(11 Suppl 1):S121-129.

7. Zhang SB, Liu TJ, Pu GH, Li BY, Gao XZ, Han XL: Suppression of Long Non-Coding RNA LINC00652 Restores Sevoflurane-Induced Cardioprotection Against Myocardial Ischemia-Reperfusion Injury by Targeting GLP-1R Through the cAMP/PKA Pathway in Mice. Cell Physiol Biochem 2018, 49(4):14761491.

8. Gao J, Xu W, Wang J, Wang K, Li P: The Role and Molecular Mechanism of Non-Coding RNAs in Pathological Cardiac Remodeling. Int J Mol Sci 2017, 18(3).

9. Wu HJ, Tang GM, Shao PY, Zou HX, Shen WF, Huang MD, Pan HH, Zhai CL, Qian G: Long non-coding RNA NEAT1 modulates hypoxia/reoxygenation-induced cardiomyocyte injury via targeting microRNA-520a. Exp Ther Med 2019, 18(3):2199-2206.

10. Kataoka M, Wang DZ: Non-Coding RNAs Including miRNAs and IncRNAs in Cardiovascular Biology and Disease. Cells 2014, 3(3):883-898.

11. Luo M, Sun Q, Zhao H, Tao J, Yan D: Long noncoding RNA NEAT1 sponges miR-495-3p to enhance myocardial ischemia-reperfusion injury via MAPK6 activation. J Cell Physiol 2020, 235(1):105-113. 
12. Yang S, Li H, Chen L: MicroRNA-140 attenuates myocardial ischemia-reperfusion injury through suppressing mitochondria-mediated apoptosis by targeting YES1. J Cell Biochem 2019, 120(3):38133821.

13. Wang DW, Lou XQ, Liu ZL, Zhang N, Pang L: LncRNA SNHG1 protects SH-SY5Y cells from hypoxic injury through miR-140-5p/Bcl-XL axis. Int J Neurosci 2020:1-10.

14. Shimokawa H, Sunamura S, Satoh K: RhoA/Rho-Kinase in the Cardiovascular System. Circ Res 2016, 118(2):352-366.

15. Cui YC, Pan CS, Yan L, Li L, Hu BH, Chang X, Liu YY, Fan JY, Sun K, Li Q et al: Ginsenoside Rb1 protects against ischemia/reperfusion-induced myocardial injury via energy metabolism regulation mediated by RhoA signaling pathway. Sci Rep 2017, 7:44579.

16. Qi Z, Li S, Su Y, Zhang J, Kang Y, Huang Y, Jin F, Xing Q: Role of microRNA-145 in protection against myocardial ischemia/reperfusion injury in mice by regulating expression of GZMK with the treatment of sevoflurane. J Cell Physiol 2019.

17. Yu SY, Tang L, Zhou SH: Long Noncoding RNAs: New Players in Ischaemia-Reperfusion Injury. Heart Lung Circ 2018, 27(3):322-332.

18. Dong Y, Chen H, Gao J, Liu Y, Li J, Wang J: Molecular machinery and interplay of apoptosis and autophagy in coronary heart disease. J Mol Cell Cardiol 2019, 136:27-41.

19. Sanz-Rosa D, Garcia-Prieto J, Ibanez B: The future: therapy of myocardial protection. Ann N Y Acad Sci 2012, 1254:90-98.

20. Xie D, Zhao J, Guo R, Jiao L, Zhang Y, Lau WB, Lopez B, Christopher T, Gao E, Cao J et al: Sevoflurane Pre-conditioning Ameliorates Diabetic Myocardial Ischemia/Reperfusion Injury Via Differential Regulation of p38 and ERK. Sci Rep 2020, 10(1):23.

21. Xie H, Zhang J, Zhu J, Liu LX, Rebecchi M, Hu SM, Wang C: Sevoflurane post-conditioning protects isolated rat hearts against ischemia-reperfusion injury via activation of the ERK1/2 pathway. Acta Pharmacol Sin 2014, 35(12):1504-1513.

22. Xu D, Dong P, Xiong Y, Yue J, Konno Y, Ihira K, Kobayashi N, Todo Y, Watari H: MicroRNA-361Mediated Inhibition of HSP90 Expression and EMT in Cervical Cancer Is Counteracted by Oncogenic IncRNA NEAT1. Cells 2020, 9(3).

23. Zhuang ST, Cai YJ, Liu HP, Qin Y, Wen JF: LncRNA NEAT1/miR-185-5p/IGF2 axis regulates the invasion and migration of colon cancer. Mol Genet Genomic Med 2020, 8(4):e1125.

24. Mou SJ, Yang PF, Liu YP, Xu N, Jiang WW, Yue WJ: BCLAF1 promotes cell proliferation, invasion and drug-resistance though targeting IncRNA NEAT1 in hepatocellular carcinoma. Life Sci 2020, 242:117177.

25. Simchovitz A, Hanan M, Niederhoffer N, Madrer N, Yayon N, Bennett ER, Greenberg DS, Kadener S, Soreq H: NEAT1 is overexpressed in Parkinson's disease substantia nigra and confers drug-inducible neuroprotection from oxidative stress. FASEB J 2019, 33(10):11223-11234.

26. Li P, Duan S, Fu A: Long noncoding RNA NEAT1 correlates with higher disease risk, worse disease condition, decreased miR-124 and miR-125a and predicts poor recurrence-free survival of acute 
ischemic stroke. J Clin Lab Anal 2020, 34(2):e23056.

27. Ren L, Chen S, Liu W, Hou P, Sun W, Yan H: Downregulation of long non-coding RNA nuclear enriched abundant transcript 1 promotes cell proliferation and inhibits cell apoptosis by targeting miR-193a in myocardial ischemia/reperfusion injury. BMC Cardiovasc Disord 2019, 19(1):192.

28. Ma M, Hui J, Zhang QY, Zhu Y, He Y, Liu XJ: Long non-coding RNA nuclear-enriched abundant transcript 1 inhibition blunts myocardial ischemia reperfusion injury via autophagic flux arrest and apoptosis in streptozotocin-induced diabetic rats. Atherosclerosis 2018, 277:113-122.

29. Huang Y: The novel regulatory role of IncRNA-miRNA-mRNA axis in cardiovascular diseases. J Cell Mol Med 2018, 22(12):5768-5775.

30. Zhao YB, Zhao J, Zhang LJ, Shan RG, Sun ZZ, Wang K, Chen JQ, Mu JX: MicroRNA-370 protects against myocardial ischemia/reperfusion injury in mice following sevoflurane anesthetic preconditioning through PLIN5-dependent PPAR signaling pathway. Biomed Pharmacother 2019, 113:108697.

31. Li H, Xu JD, Fang XH, Zhu JN, Yang J, Pan R, Yuan SJ, Zeng N, Yang ZZ, Yang H et al: Circular RNA circRNA_000203 aggravates cardiac hypertrophy via suppressing miR-26b-5p and miR-140-3p binding to Gata4. Cardiovasc Res 2020, 116(7):1323-1334.

32. Wang S, Cui Y, Xu J, Gao H: miR-140-5p Attenuates Neuroinflammation and Brain Injury in Rats Following Intracerebral Hemorrhage by Targeting TLR4. Inflammation 2019, 42(5):1869-1877.

33. Zou G, Zhong W, Wu F, Wang X, Liu L: Catalpol attenuates cardiomyocyte apoptosis in diabetic cardiomyopathy via Neat1/miR-140-5p/HDAC4 axis. Biochimie 2019, 165:90-99.

34. Luo SY, Chen S, Qin YD, Chen ZW: Urotensin-Receptor Antagonist SB-710411 Protects Rat Heart against Ischemia-Reperfusion Injury via RhoA/ROCK Pathway. PLoS One 2016, 11(1):e0146094.

35. Pearn ML, Schilling JM, Jian M, Egawa J, Wu C, Mandyam CD, Fannon-Pavlich MJ, Nguyen U, Bertoglio J, Kodama $\mathrm{M}$ et al: Inhibition of RhoA reduces propofol-mediated growth cone collapse, axonal transport impairment, loss of synaptic connectivity, and behavioural deficits. $\mathrm{Br} \mathrm{J}$ Anaesth 2018, 120(4):745-760.

36. Gong P, Li R, Jia HY, Ma Z, Li XY, Dai XR, Luo SY: Anfibatide Preserves Blood-Brain Barrier Integrity by Inhibiting TLR4/RhoA/ROCK Pathway After Cerebral Ischemia/Reperfusion Injury in Rat. J Mol Neurosci 2020, 70(1):71-83.

37. Forgione N, Fehlings MG: Rho-ROCK inhibition in the treatment of spinal cord injury. World Neurosurg 2014, 82(3-4):e535-539.

\section{Tables}

Table 1 Primers for reverse transcription quantitative polymerase chain reaction 
Targets Primer sequence (5'-3')

NEAT1 Forward: CAAATTCGTGAAGCGTTCCATA

Reverse: GTGCCGAGTCCGGACGATATTC

miR-140 Forward: TACCACAGGGTAGAACCACGG

Reverse: TGCTTGGCAATAACAGACCAAC

RhoA Forward: GATTGGCGCTTTTGGGTACAT

Reverse: AGCAGCTCTCGTAGCCATTTC

U6 Forward: CTCGCTTCGGCAGCACA

Reverse: AACGCTTCACGAATTTGCGT

GAPDH Forward: GGAGCGAGATCCCTCCAAAA

Reverse: GGCTGTTGTCATACTTCTCATGG

Note: NEAT1, nuclear enriched abundant transcript 1; miR-140, microRNA-140; GAPDH, glyceraldehyde-3-phosphate dehydrogenase

Figures 
A

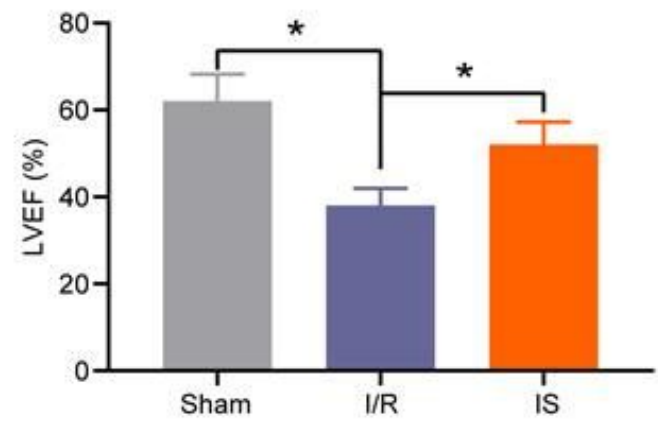

B
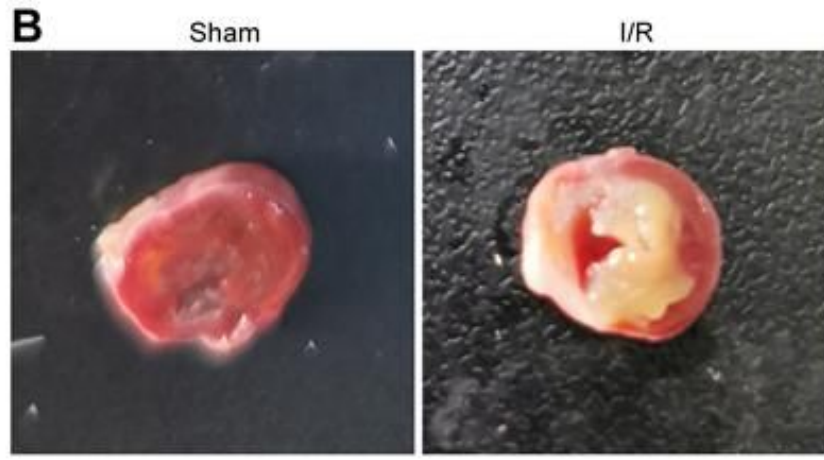

C

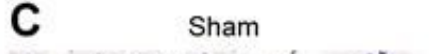

Sham

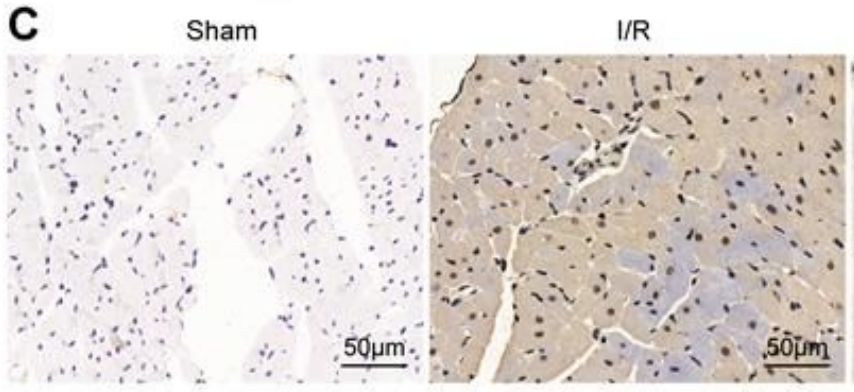

50jm :
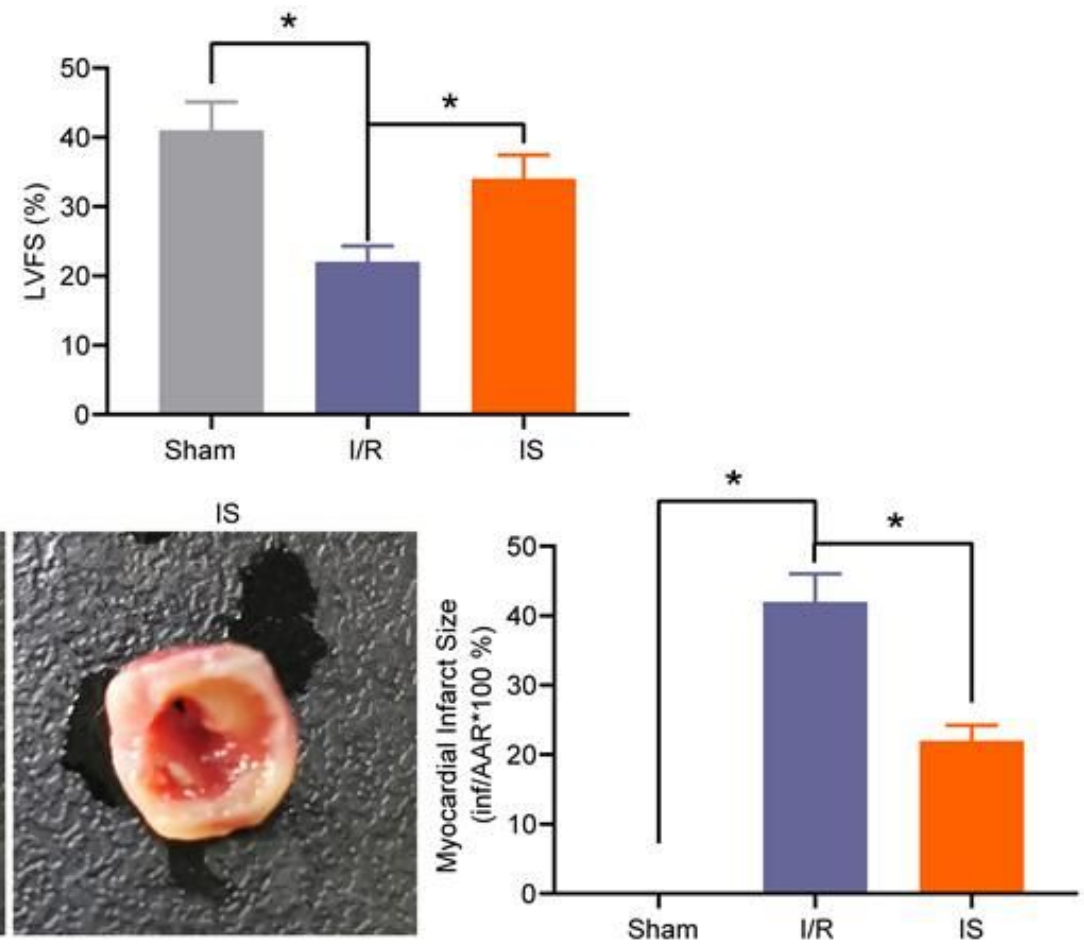

IS
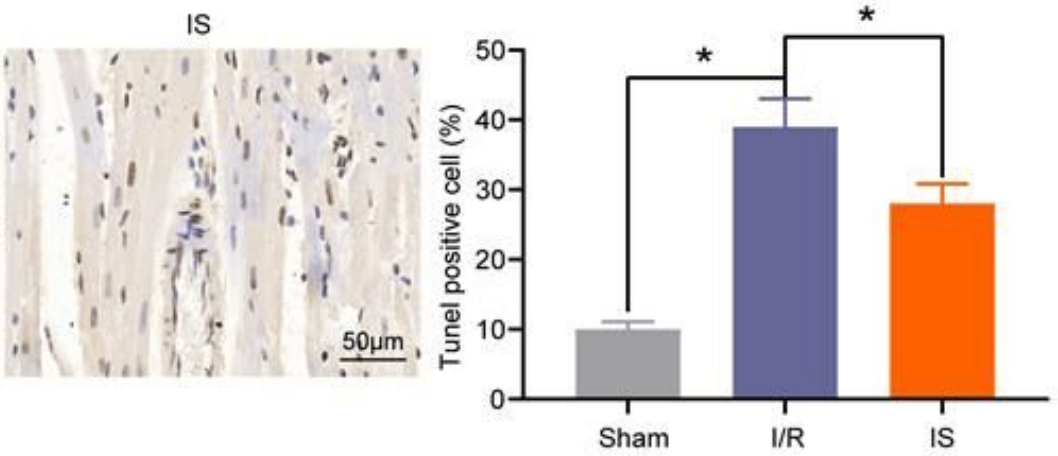

Figure 1

Sev acts as a cardio-protector in mice with injury caused by myocardial I/R. A, LVEF and LVFS in mice with I/R injury and Sev-treated model evaluated by ECG; B, myocardial infarct area in mice tested by TTC staining; $C$, the apoptosis rate of $\mathrm{I} / \mathrm{R}$ and Sev treated model assessed by TUNEL staining. ${ }^{*} p<0.05$ according to the one-way ANOVA. Results are expressed as mean $\pm S D(n=3$ per group). Experiment was repeated 3 times. 

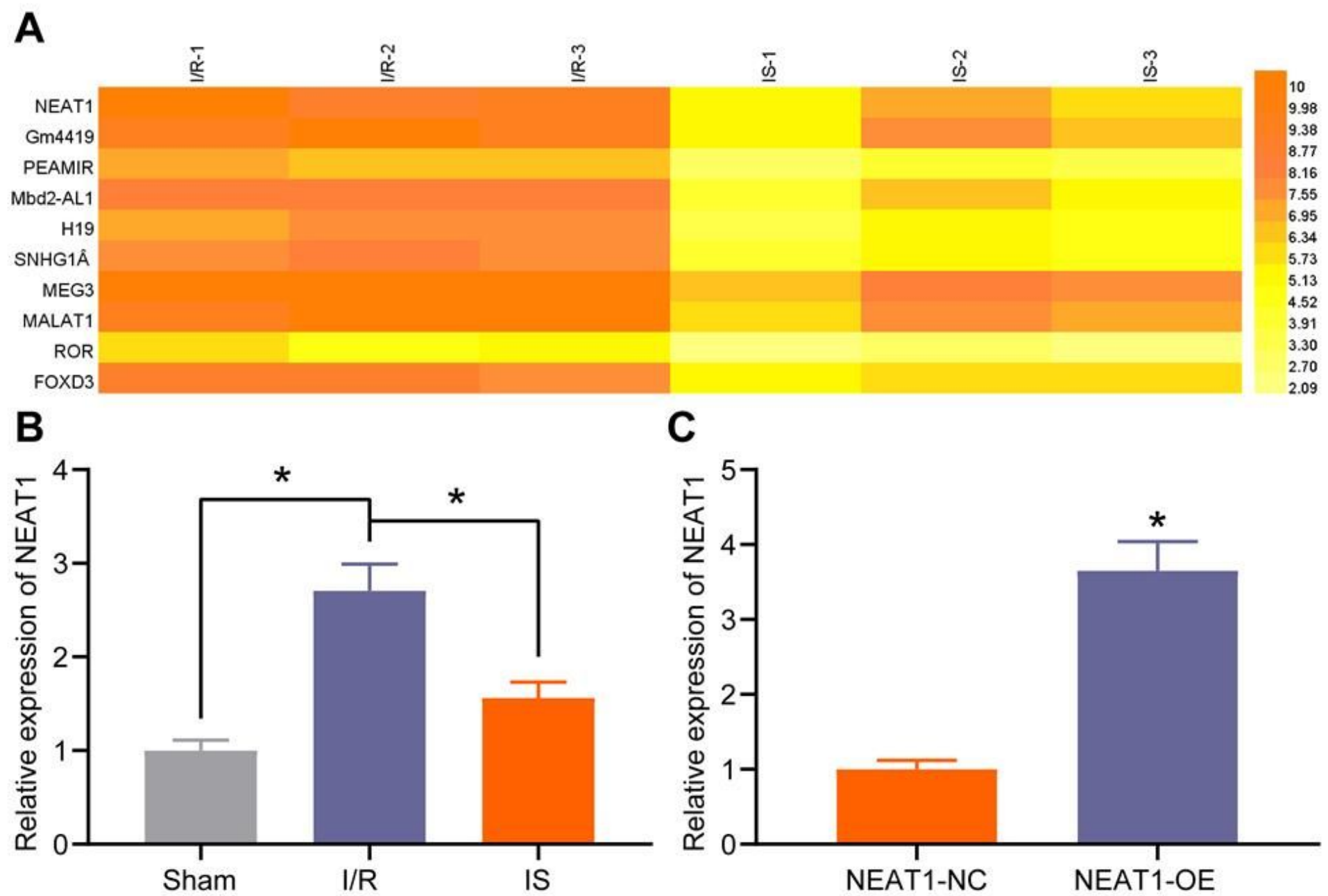

Figure 2

NEAT1 is downregulated after Sev treatment in mice with myocardial I/R. A, differentially expressed IncRNAs between mice subjected to I/R injury with or without Sev treatment screened out by microarray analysis; B, NEAT1 expression in mice under different treatments evaluated by RT-qPCR; C, NEAT1 expression in Sev-injected mice administrated with NEAT1-OE or NEAT1-NC. In panel B, * $p<0.05$ according to the one-way ANOVA; In panel $\mathrm{C},{ }^{*} \mathrm{p}<0.05$ according to unpaired $t$ test. Results are expressed as mean $\pm S D$ ( $n=3$ per group). Experiment was repeated 3 times. 

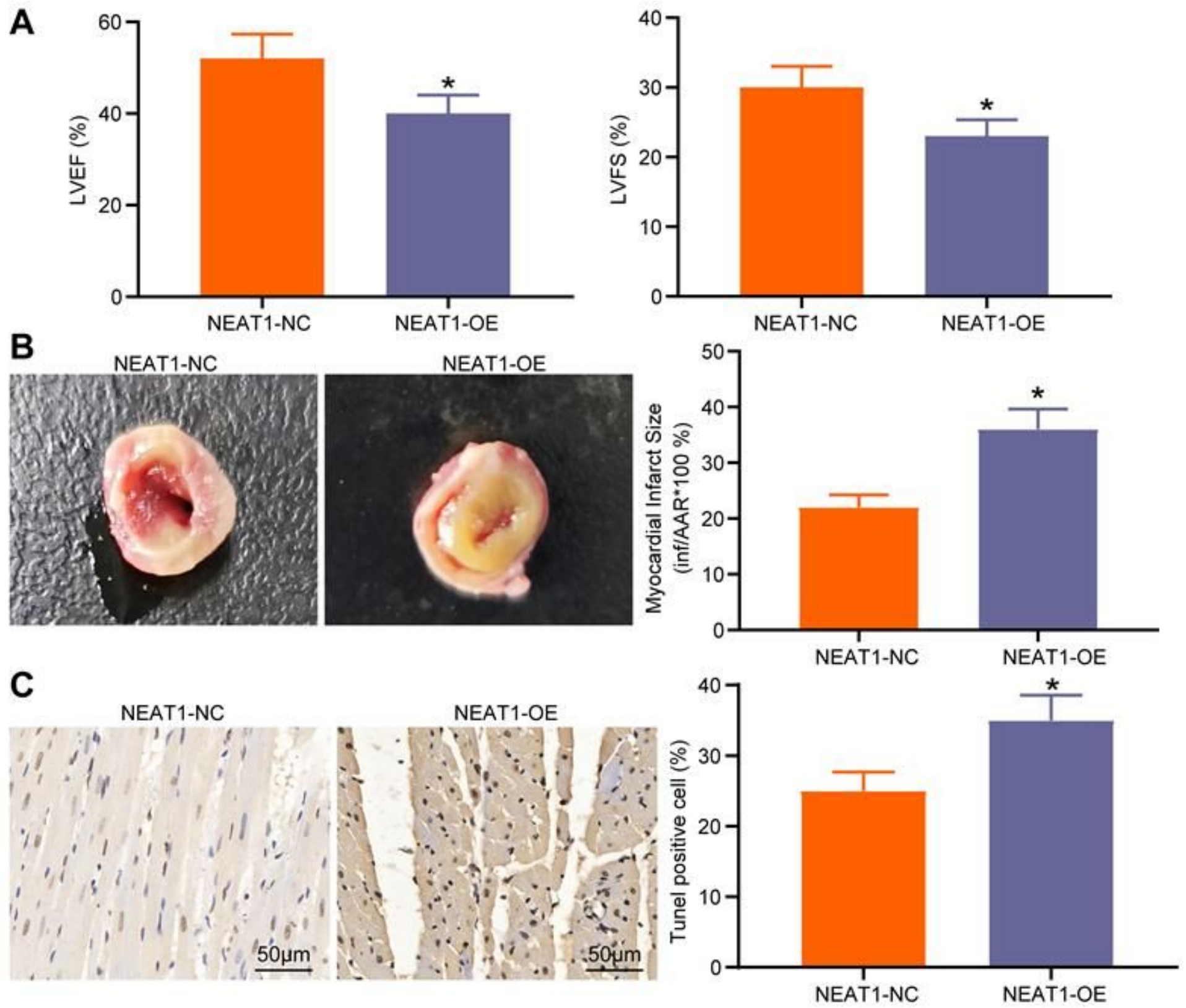

\section{Figure 3}

NEAT1 upregulation weakens the cardioprotective role of Sev on mice with myocardial I/R injury. A, LVEF and LVFS in mice injected with NEAT1-OE or NEAT1-NC evaluated by ECG; B, myocardial infarct area in mice injected with NEAT1-OE or NEAT1-NC tested by TTC staining; $C$, the apoptosis rate of injected with NEAT1-OE or NEAT1-NC-treated model assessed by TUNEL staining. ${ }^{*} \mathrm{p}<0.05$ according to the unpaired $t$ test. Results are expressed as mean \pm SD ( $n=3$ per group). Experiment was repeated 3 times. 


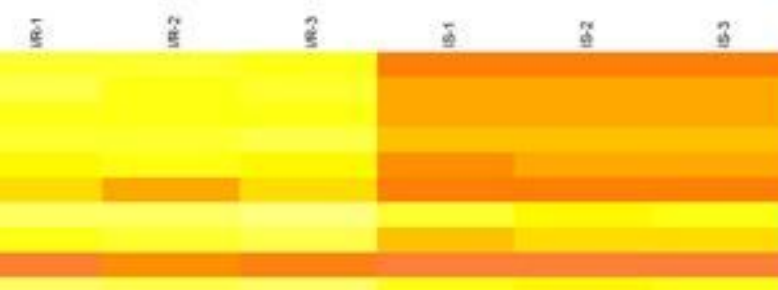

B

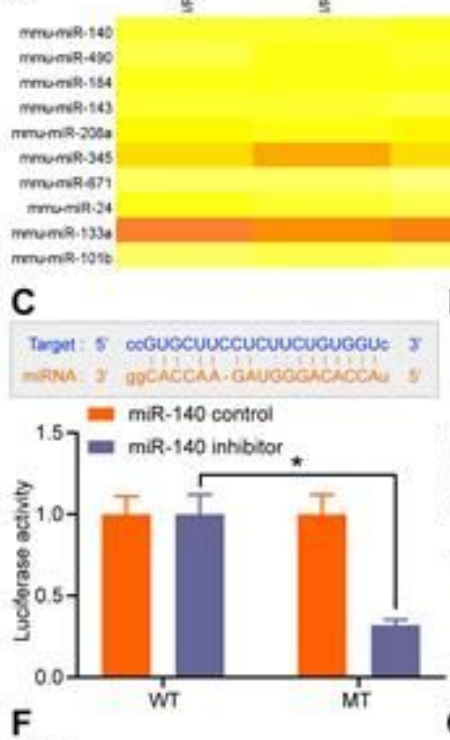

D

E
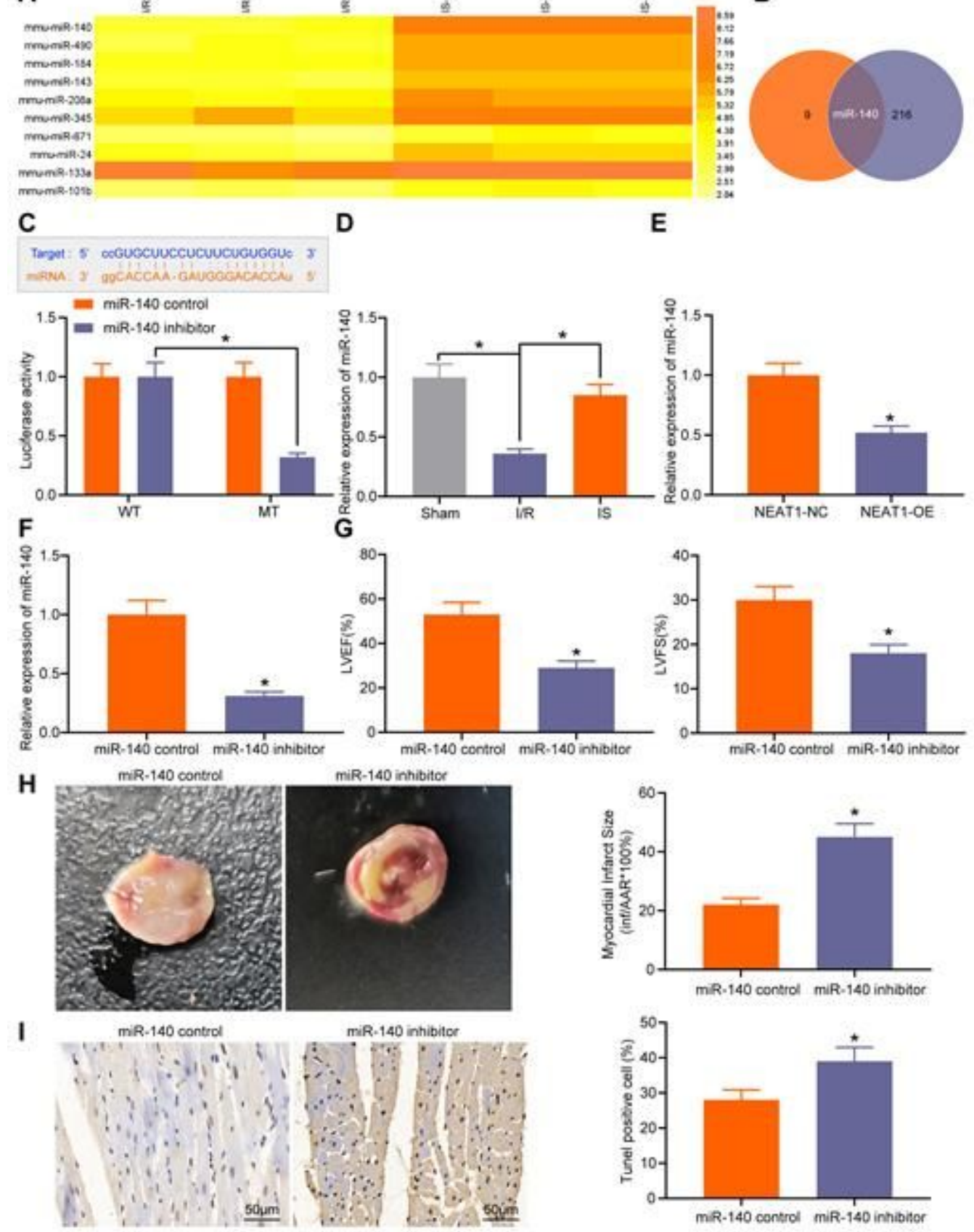

Figure 4

miR-140, restored after Sev treatment, is involved in the modulation of Sev-alleviated myocardial I/R injury. A, differentially expressed miRNA between mice subjected to I/R injury with or without Sev treatment screened out by microarray analysis; B, the intersected miRNA in microarray and StarBase prediction results; $\mathrm{C}$, the binding relationship between miR-140 and NEAT1 predicted by StarBase and validated by a dual luciferase reporter gene assay; D, miR-140 expression in mice under different treatments evaluated by RT-qPCR; E, miR-140 expression in Sev-injected mice administrated with NEAT1OE or NEAT1-NC; F, miR-140 expression in Sev-injected mice administrated with miR-140 inhibitor or miR140 control; G, LVEF and LVFS in mice injected with miR-140 inhibitor or miR-140 control evaluated by ECG; $\mathrm{H}$, myocardial infarct area in mice injected with miR-140 inhibitor or miR-140 control tested by TTC 
staining; I, the apoptosis rate of injected with miR-140 inhibitor or miR-140 control-treated model assessed by TUNEL staining. In panel $C$, ${ }^{*} p<0.05$ according to the two-way ANOVA; In panel $D-I,{ }^{*} p<0.05$ according to unpaired $t$ test. Results are expressed as mean $\pm S D$ ( $n=3$ per group). Experiment was repeated 3 times.
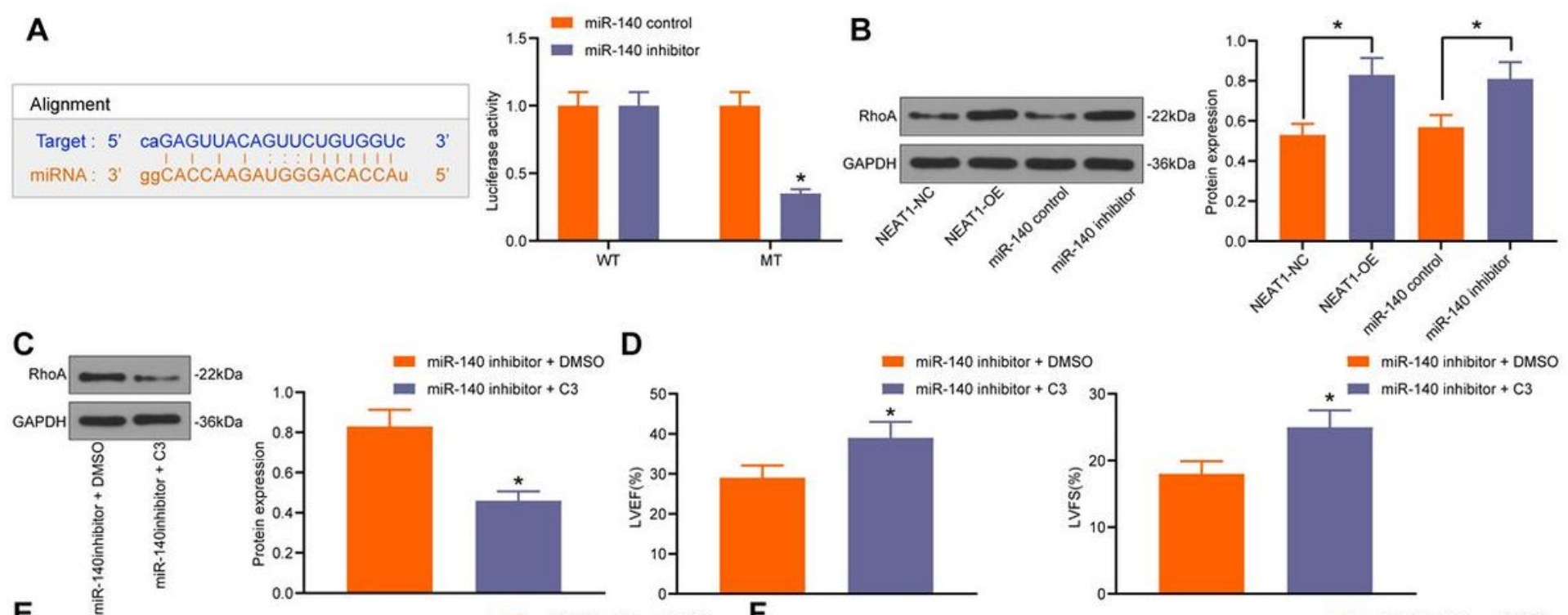

D
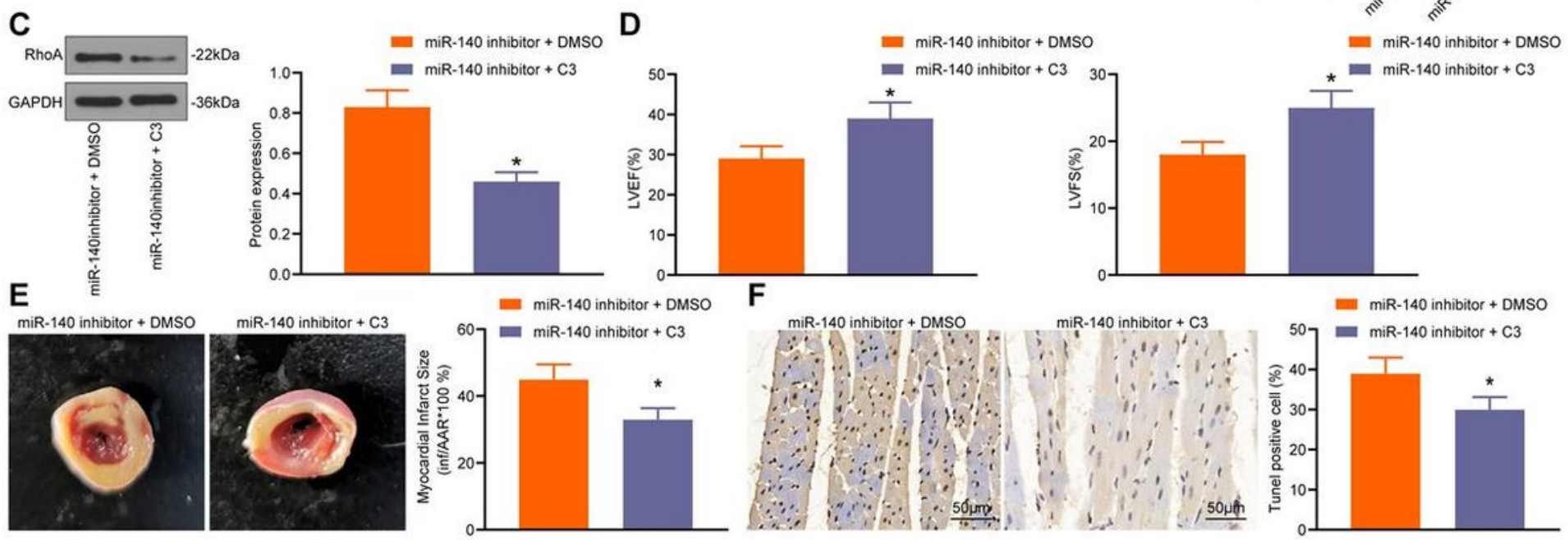

$\mathbf{F}$

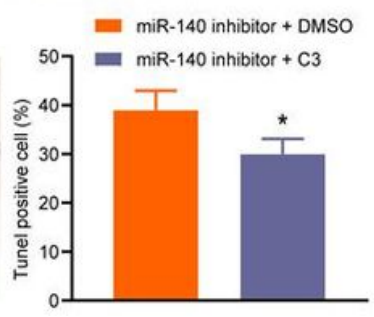

\section{Figure 5}

RhoA, a target of miR-140, is involved in the modulation of miR-140-dependent myocardial I/R injury. A, the binding relationship between miR-140 and RhoA predicted by StarBase and validated by a dual luciferase reporter gene assay; $B$, the protein expression of RhoA in nude mice injected with NEAT1-NC, NEAT1-OE, miR-140 control and miR-140 inhibitor determined by western blot analysis; C, the protein expression of RhoA in nude mice injected with miR-140 inhibitor + C3 or miR-140 inhibitor + DMSO determined by western blot analysis; D, LVEF and LVFS in mice injected with miR-140 inhibitor + C3 or miR-140 inhibitor + DMSO evaluated by ECG; E, myocardial infarct area in mice injected with miR-140 inhibitor + C3 or miR-140 inhibitor + DMSO tested by TTC staining; F, the apoptosis rate of injected with miR-140 inhibitor + C3 or miR-140 inhibitor + DMSO-treated model assessed by TUNEL staining. In panel A-C, ${ }^{*} p<0.05$ according to the two-way ANOVA; In panel $D-F,{ }^{*} p<0.05$ according to unpaired $t$ test. Results are expressed as mean $\pm S D$ ( $n=3$ per group). Experiment was repeated 3 times. 


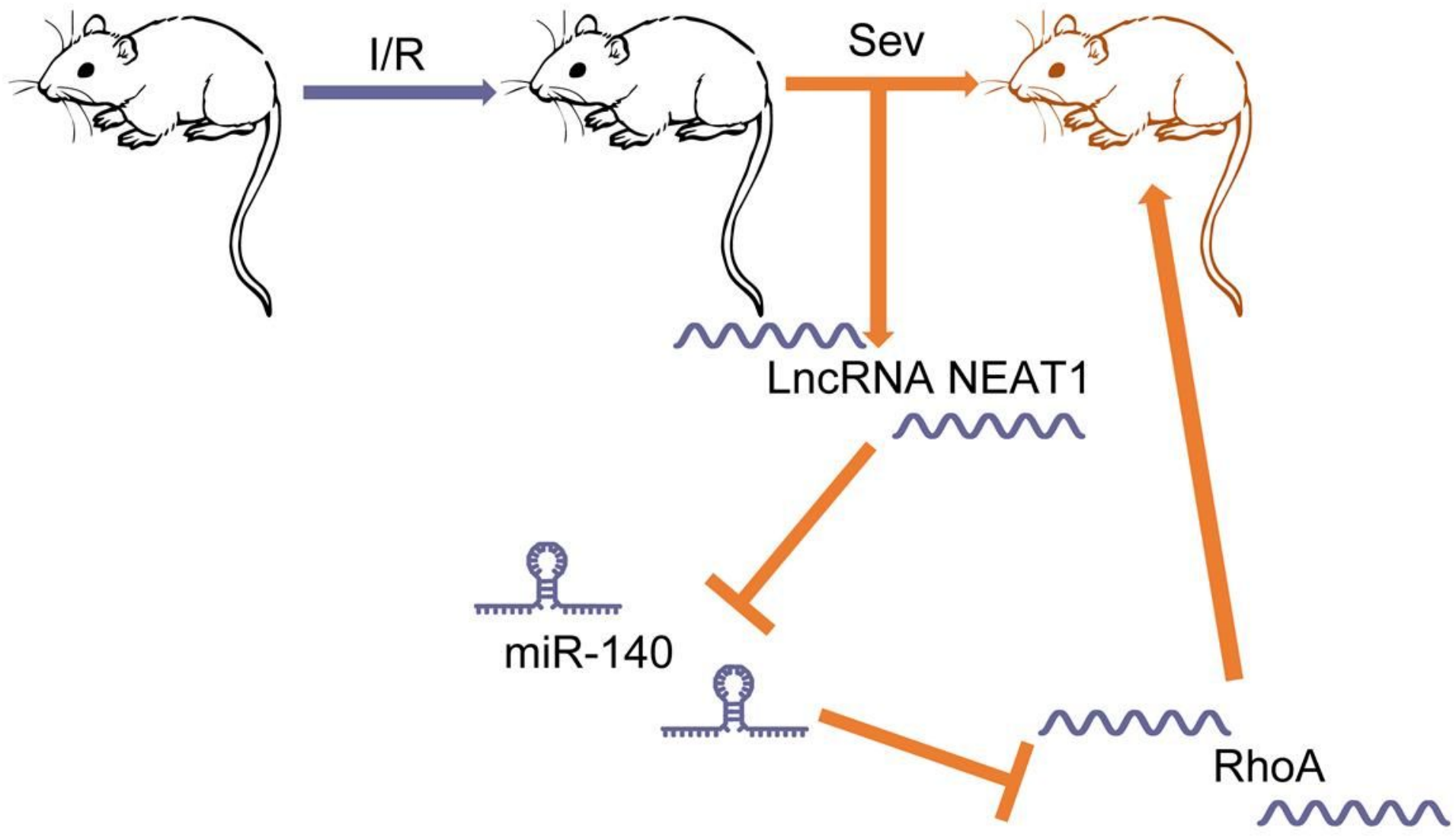

\section{Figure 6}

A diagram illustrating Sev diminishes injury caused by myocardial I/R via the NEAT1/miR-140/RhoA axis. NEAT1 sponged miR-140 to mediate RhoA expression. Sev treatment mitigated I/R injured mice by downregulating NEAT1 and RhoA, while upregulating miR-140 expression.

\section{Supplementary Files}

This is a list of supplementary files associated with this preprint. Click to download.

- ARRIVEchecklist.docx

- Originallmages.docx 